\title{
Rating Methodology, Personality Axes, and Behavioral Plasticity: A Case Study in African Lions
}

\author{
Lauri L. Torgerson-White ${ }^{1 *}$ and Cynthia Bennett ${ }^{2}$
}

\begin{abstract}
${ }^{1}$ Detroit Zoological Society
${ }^{2}$ Journal for Applied Animal Welfare Science

*Corresponding author (Email: 1torgerson@dzs.org)

Citation - Torgerson-White, L. L., \& Bennett, C. (2014). Rating methodology, personality axes, and behavioral plasticity: A case study in African lions. Animal Behavior and Cognition, 1(3), 230-248. doi: 10.12966/abc.08.02.2014
\end{abstract}

\begin{abstract}
Animal personality refers to individual differences in behavior that are consistent across time and across contexts. As it plays an ecological and evolutionary role for many animals by affecting fitness, it is likely to play a role in how both species and individuals fare in captivity. Assessment of animal personality by caretaker ratings has been validated using behavioral coding methods in a number of species and has thus become the most common method for assessing personality of captive animals. Whereas the reliability of rating methodology is arguable, the reality of working in a captive setting makes assessment of animal personality by caretaker ratings an easy and practical way to investigate questions related to personality. We used a case study in African lions to investigate the use of rating methodology to uncover personality traits. We then related dimensionality of those traits to fecal glucocorticoid metabolite values taken before, during, and after habitat renovation. The end goal was to devise a method by which individual welfare can be considered and improved by considering personality traits. Interobserver reliability scores revealed that only 11 out of 18 traits and four out of six lions were reliably rated. From those traits, we uncovered a sociability-neuroticism axis, whereby lions that were more social and less neurotic had lower glucocorticoid metabolite levels (GCM). In addition, we found that more vocal animals had higher GCM levels and more active animals had lower GCM levels, findings that suggest that vocalizing and activity level may be part of the way these individuals cope with stressors. These results suggest that a better understanding of a lion's sociability and neuroticism may inform management decisions during stressful times. Further investigation into the two lions who did not pass reliability testing, revealed that they had the lowest number of extreme trait ratings which suggests that they may have been the most behaviorally plastic, a characteristic that was not expressly measured by the surveys. The inability of rating methodology to measure plasticity and the role of the human-animal relationship in contributing to low reliability scores are discussed. We suggest that the most widely used personality surveys may work well for animals who exhibit low levels of plasticity and whose behavior falls closer to the extremes of a continuum, but may be less helpful for more plastic animals whose personality traits fall in the mid-range.
\end{abstract}

Keywords - Personality, Temperament, Behavioral syndrome, Coping style, Inter-observer reliability, African lions, Glucocorticoids, Animal welfare, Behavioral plasticity, Stress

Animal welfare is an animal's collective physical, mental, and emotional state over a period of time, and is measured on a continuum from good to poor (Association of Zoos and Aquariums Animal Welfare Committee, 2014). By definition, it is an attribute of an individual animal. For that reason, when trying to improve welfare, captive animal management decisions should be made at the level of the individual, as well as at the group or species level. Many individual factors are utilized when making these decisions, including the animal's sex, age, health status, social status, and often, personality (Coleman, 2012; Watters \& Powell, 2012; Whitham \& Wielebnowski, 2009). 
The term personality refers to individual behavioral differences that are consistent across time and across contexts (Budaev, Zworykin, \& Mochek, 1999). When deriving personalities, several behaviors or personality traits are empirically measured and then combined into personality axes using multivariate statistical techniques, most commonly principal component analysis (PCA) or factor analysis. These axes vary along particular dimensions such as shyness/boldness and social/anti-social, among others (Réale, Reader, Sol, McDougall, \& Dingemanse, 2007; Sih, Bell, \& Johnson, 2004; Sih, Bell, Johnson, \& Ziemba, 2004). Individuals can then be assessed regarding where they fall along various behavioral traits that constitute these personality axes. Distinct personalities, also known as temperaments or behavioral syndromes, have been validated across numerous species and have been shown to be heritable and adaptive in nature (Biro \& Stamps, 2008; Dingemanse \& Réale, 2005; Réale et al., 2007; Sih, Bell, \& Johnson, 2004; Sih, Bell, Johnson, et al., 2004; Vazire, Gosling, Dickey, \& Schapiro, 2007; Wolf, van Doorn, Leimar, \& Weissing, 2007). For example, exploratory tendency is heritable and adaptive in great tits (Parus major), with survival among the different phenotypes varying with the amount of food available (Dingemanse \& Réale, 2005). The impact of personality is likely not diminished when animals are moved into captivity. It is known that certain species fare better in captivity than others and that the existence of species-typical personality traits is one of many factors that likely contribute to the degree of welfare experienced by different species. Specifically, it has been suggested that boldness and behavioral flexibility may predict the level of welfare experienced by captive species (Mason, 2010). If personality traits affect individual fitness in wild animals as well as welfare at the species level in captivity, it follows that personality should impact individual welfare of captive animals. Specifically, personality traits that help individuals better cope with captive life should be favored, thus influencing the proportion or diversity of those traits present in the captive population (McDougall, Réale, Sol, \& Reader, 2006).

Because some animals have physiological reactions to stress that do not manifest themselves as clear behaviors (Koolhaas et al., 1999), to gain a more complete understanding of how an animal copes with its environment, one should investigate both behavioral and physiological measures of welfare. Zoo researchers often utilize glucocorticoid measures as a way to assess how animals cope with captive stressors (Armstrong \& Santymire, 2013; Brown, Kersey, Freeman, \& Wagener, 2010; Burgener, Gusseta, \& Schmidb, 2008; Loeding, Thomas, Bernier, \& Santymire, 2011). A link between personality measures (behavioral responses that are consistent across time) and the physiological stress response (i.e., the hypothalamic-pituitary-adrenal (HPA)) axis activity) has been termed a coping style (Koolhaas et al., 1999). Research has revealed that many species have a proactive-reactive coping style (Sih, Bell, \& Johnson, 2004). Proactive individuals are more aggressive, less flexible, have low HPA axis activity, and fare better in stable environments while reactive individuals are less aggressive, more flexible, have high HPA axis activity, and fare better in changing environments (Koolhaas et al., 1999; Sih, Bell, Johnson, et al., 2004). As glucocorticoids or behavior alone are sometimes used to assess welfare in captivity, it is becoming increasingly important to validate objective measures of personality and to link them to HPA axis reactivity in captive animals.

Considering personality when making animal management decisions is not new. It is a common, if informal, practice that usually uses a subjective assessment of personality as given by the caretaker(s) who knows the animal best. In recent years, it has become apparent that a scientific approach to measuring animal personality needs to be taken (Gosling \& John, 1999; Réale et al., 2007; Sih, Bell, \& Johnson, 2004). The methodology used to measure personality in captive animals generally falls into two categories, ratings and coding. The rating method relies on the ability of individuals who know the animals well to score or rate the animals along a continuum of highest to lowest on multiple behavioral traits. These ratings are quantifiable and can then be analyzed to extract personality axes. Limitations of this method include sensitivity to the nature of the rater's relationship with the individual animals and a failure to capture behavioral plasticity (Highfill, Hanbury, Kristiansen, Kuczaj, \& Watson, 2010; Réale \& Dingemanse, 2012). The coding method utilizes behavioral data collection techniques to observe the frequency or duration of a pre-determined list of behaviors in the animals in order to create behavioral time budgets. It is from these behavioral data that personality axes are then extracted using multivariate 
statistical techniques. Because of the abundance of individuals who know the animals well and the ease with which personality surveys can be administered, the rating method is the most popular method by which zoos assess animal personality (Watters \& Powell, 2012).

Previous research on personality in African lions utilized rating methodology based on multiple personality surveys to derive three personality axes: dominance, impulsiveness, and neuroticism (Gartner, Powell, \& Weiss, in press). Dominant individuals rated highly on the traits jealous, stingy, and aggressive to conspecifics. Impulsive individuals rated highly on the traits active, erratic, and eccentric. Finally, neurotic individuals rated highly on the traits fearful of people, insecure, and tense. Because lions are one of only a few cat species considered to be predominantly social, we suggest that several of the traits that occur in these axes, specifically those that measure fear of people or conspecifics and aggression or friendliness towards people or conspecifics, may also explain the individual's level of sociability, or preference for being with others rather than being alone (Cheek \& Buss, 1981).

Understanding how personality might influence the ability of an animal to cope with environmental stressors may enable individualized animal management during potentially stressful events and thus, improve animal welfare. In 2011, the Detroit Zoo renovated its lion habitat, with the goal of increasing the space available to the animals. During this change, we attempted to validate a caretaker rating survey using glucocorticoid metabolite (GCM) measures with the objectives being to uncover coping styles in these lions and thus predict in the future how these animals would cope with environmental stressors. Before, during, and after construction, fecal samples were collected that would later be analyzed for fecal glucocorticoid metabolites. Glucocorticoid metabolite levels were compared with behavioral data taken from caretaker logs in order to determine if high GCM levels were indicative of stress. Because we had access to three caretakers who all had significant experience with the animals, the rating method was used to measure individual personality traits in each of the animals. An attempt was then made to relate these personalities to the glucocorticoid metabolite levels observed in the lions before, during, and after habitat modification. We will discuss our results as well as reasons why this case study led us to the conclusion that extreme care needs to be taken when using caretaker ratings to measure animal personality.

\section{Method}

\section{Animals and Facilities}

Study animals were six adult African lions (Panthera leo senegalensis) (2 males; 4 females) (mean age: $15.1 \pm 1.6$ year; range: 11-18 years) housed in the Detroit Zoo's African Grasslands habitat, which underwent renovation from March until August 2011. Over the course of this study the habitat was enlarged from $334 \mathrm{~m}^{2}$ to $771 \mathrm{~m}^{2}$. Both the old and the renovated habitats had grassy substrates, deadfall logs, and concrete climbing structures. Heated rocks and a water feature were added after construction and containment was changed from a moated exhibit to one where a transparent barrier and low fencing separated the animals from the public. The animals were managed in three social groupings (Erin, Emily, and Larson; Percival and Bikira; Katie and Bikira) and were separated for feedings. The lions were fed Natural Balance ${ }^{\circledR}$ Zoo Carnivore diet until August $15^{\text {th }}$, when they completed a transition to Nebraska ${ }^{\circledR}$ classic feline diet (except Percival, who was switched to Nebraska ${ }^{\circledR}$ senior feline diet). They were fasted on Saturdays, with bones given Monday, Wednesday, and Saturday. Water was offered ad libitum. Before and after construction, social groups were rotated between the indoor holding areas and the outdoor habitat on a 24-hr cycle. During construction, the lions were housed inside in the same social groups. Inside there were a total of ten stalls configured in two rows along both sides of a single, wide aisle way. Four stalls were on one side of the aisle way and six on the other. A visual barrier was used to prevent animals housed on different sides of the aisle way from seeing one another. Stalls varied in size from 28 to $35 \mathrm{~m}^{2}$. Each had a raised Lexan ${ }^{\circledR}$ bed. Transfer doors between the stalls were kept open to provide access to as many stalls as possible. Over the course of the study, there were no social disruptions or staff changes. Management and husbandry routines remained consistent. 


\section{Hormone Analysis}

Fecal samples were identified individually by color (animals were fed a small bolus of their regular diet into which a small quantity of uncooked rice dyed with food coloring was mixed) and were collected once a day at approximately the same time of day, as often as possible during the study. Each sample was placed in a sealable plastic bag and frozen at -20 degrees Celsius until glucocorticoid metabolite analysis in November 2012. A total of 225 samples collected two weeks prior to the beginning of construction (March 7-March 21), four weeks following the start date of construction (March 22-April 20), and four weeks following release into the new habitat (August 9-September 24, these dates varied somewhat for the different social groups) were analyzed for fecal glucocorticoid metabolites by the St. Louis Zoo Endocrinology Laboratory. On average, 2.8 samples were collected and analyzed per lion per week, for a total of 37.5 samples per lion.

Fecal extraction. Approximately $0.5 \mathrm{~g}$ of fecal material was shaken overnight in $5 \mathrm{~mL}$ modified phosphate-saline buffer containing 50\% methanol, 0.1\% BSA and 5\% Tween 20, a modification of the method of Shideler et al. (Shideler, Ortuno, Moran, Moorman, \& Lasley, 1993). Solids were removed by centrifugation, and the supernatants stored at $-70^{\circ} \mathrm{C}$ until assay.

Glucocorticoid metabolite assay. Fecal corticoid levels were determined using a commercially available corticosterone radioimmunoassay (DA Corticosterone kit, ICN MP Biomedicals). Although glucocorticoids are the primary circulating corticoid in the blood of most mammals, they are excreted as a mixture of corticoid metabolites. This particular assay has been shown to cross-react with glucocorticoid metabolites in a wide variety of species (Wasser et al., 2000).

The lower detection limit was $0.625 \mathrm{ng} / \mathrm{ml}$ and upper detection limit was $50 \mathrm{ng} / \mathrm{ml}$. Assays were performed according to manufacturer's protocols with the exception that the matrices were equalized by adding standard diluent to the fecal extracts and fecal extraction buffer (containing $50 \%$ methanol) to the standards. Concentrations were determined as $\mathrm{ng} / \mathrm{ml}$, and then divided by the dry weight of feces extracted to give the results as ng/g feces. All samples were assayed in duplicate. The assay was repeated for any sample in which the duplicates differed by $15 \%$ or greater. Mean intra-assay variation of duplicate samples was $6.97 \%$. Mean inter-assay coefficients of variation for low and high quality controls were $10.9 \%$ and $9.3 \%$ respectively.

Assay validation. Fecal extracts were tested for linearity by diluting six samples that contained high levels of hormone by $1 / 2,1 / 4,1 / 8$, and $1 / 16$ with extraction buffer. Serial dilutions of lion fecal extracts measured an average of $103.45 \pm 0.27 \%$ of expected values. We assessed the accuracy of the assay by adding a known amount of corticosterone to six fecal extracts containing low values of corticosterone. Addition of known amounts of hormone at three dosage levels resulted in recovery of $95.34 \pm 0.91 \%$ of added corticosterone.

\section{Personality Assessment}

We utilized a personality survey that was based on adjectives used to describe domestic cats and was previously validated in captive cheetahs (Wielebnowski, 1999). The survey asked caretakers to measure the following traits: active, aggressive to conspecifics, aggressive to people, calm, curious, eccentric, excitable, friendly to conspecifics, friendly to people, fearful of conspecifics, fearful of people, insecure, playful, self-assured, smart, solitary, tense, and vocal (Table 1). The survey included the 18 behavioral traits listed above, with a $120 \mathrm{~mm}$ line drawn under each trait that represented a continuous scale for that trait. Caretakers were asked to place a tick mark on each of the horizontal lines, with the placement indicating how much each particular adjective applied to the lion in question, in comparison to all lions they had ever known. For example, when assessing activity, a mark placed all the way to the left would indicate that the lion in question was the least active lion they had ever known. A mark placed all the way to the right would indicate that the lion in question was the most active lion they had ever known. 
Table 1

\begin{tabular}{|c|c|}
\hline Trait & Definition \\
\hline Active & Moves frequently (e.g., paces, runs, stalks a lot). \\
\hline $\begin{array}{l}\text { Aggressive to } \\
\text { conspecifics }\end{array}$ & Frequently reacts in a hostile manner (e.g., attacks, growls) toward other lions. \\
\hline $\begin{array}{l}\text { Aggressive to } \\
\text { people }\end{array}$ & Frequently reacts in a hostile or threatening manner towards people. \\
\hline Calm & Not easily disturbed by changes in the environment. \\
\hline Curious & Readily approaches and explores changes in the environment. \\
\hline Eccentric & Shows stereotypic or unusual behaviors. \\
\hline Excitable & Overreacts to changes in the environment. \\
\hline $\begin{array}{l}\text { Friendly to } \\
\text { conspecifics }\end{array}$ & Social; initiates and seems to seek closer proximity to other lions. \\
\hline Friendly to people & $\begin{array}{l}\text { Initiates closer proximity; approaches mesh readily and in a friendly manner (e.g., purrs, rubs } \\
\text { on mesh). }\end{array}$ \\
\hline $\begin{array}{l}\text { Fearful of } \\
\text { conspecifics }\end{array}$ & Retreats and hides readily from other lions. \\
\hline Fearful of people & Retreats readily from people. \\
\hline Insecure & Seems scared easily; "jumpy" and fearful in general. \\
\hline Playful & $\begin{array}{l}\text { Initiates and engages in play behavior (seemingly meaningless, but non-aggressive behavior) } \\
\text { with objects and/or other lions. }\end{array}$ \\
\hline Self-assured & Moves in a seemingly confident, well-coordinated, and relaxed manner. \\
\hline Smart & Learns quickly to associate certain events and appears to remember for a long time. \\
\hline Solitary & Spends time alone; avoids company. \\
\hline Tense & Shows restraint in movement and posture. \\
\hline Vocal & Frequently and readily vocalizes. \\
\hline
\end{tabular}

Three caretakers who had worked with these lions from three to 13 years $(x=7.1 \pm 3.6)$ rated the lions. One caretaker cared for the lions five days per week, the second two days per week, and the third did enrichment and training, with the number of days per week for care being variable. All three caretakers fed and enriched the lions and participated in shifting and cleaning. The caretaker who cared for the lions two days per week was involved in substantially less training than the other two. Only the five-day caretaker was involved in veterinary procedures (with the exception of blood draw training). Each of the caretakers had cared for between 11 and 15 lions in their careers. The instructions were verbally explained to each caretaker, who had an opportunity to ask questions and was instructed not to communicate with the other caretakers regarding the survey. Each caretaker was asked to fill out one survey per lion. 
Torgerson-White \& Bennett 235

\section{Statistical Analysis}

Glucocorticoid metabolite levels across conditions. A linear mixed effect model ( $\mathrm{R}$ version 3.0.3, package lme4) investigated the impact of condition (before, during, or after construction) as a fixed effect and individual lion (ID) as a random effect on log-transformed GCM levels ( $n=6$ lions). In order to assess the relevance of ID, this model was then compared to a model without ID as a random effect by computing the likelihood ratio test statistic and its corresponding $p$-value (Faraway, 2006). Individual differences in log-transformed GCM levels across conditions were then assessed separately for each lion using analysis of variance.

Inter-observer reliability testing. Many zoo personality studies test inter-observer reliability (IOR) on either the traits or the animals (Phillips \& Peck, 2007; Wielebnowski, 1999). Presumably, it is assumed that testing IOR on trait ratings will account for any disagreement on individual animals, and vice versa. We decided to test this assumption by first testing IOR on traits, removing those traits that did not pass reliability testing, and then testing IOR on the animals. If testing IOR on traits should account for disagreement on individual animals, then it follows that all animals should pass reliability testing after the unreliable traits are removed. Thus, inter-observer reliability of all traits was assessed using Kendall's coefficient of concordance, W (Siegel \& Castellan, 1988). Because of the small sample size $(n=6)$ and because concordance values were generally high, a significance level of $p=0.1$ was used (Wielebnowski, 1999). Traits for which reliability was below $\mathrm{W}=0.6$, the point at which the $p$ value fell below 0.1 , were removed from further analyses. After removing the unreliable traits, IOR was then assessed for each lion and lions for which reliability was below $\mathrm{W}=0.6$ were removed from the PCA and any models that included PC scores.

Extracting personality axes. Principal component analysis was used to reduce the dimensionality of the dataset by combining the original variables into a reduced number of orthogonal variables that represent the maximum variability of the covariance structure of the data (Johnson \& Wichern, 2002). In this case, PCA was used to extract a personality axis from the 11 reliable behavioral traits for the four reliably rated lions. First, trait values for the 11 reliable traits, averaged across all three caretakers, were loaded into a PCA using the correlation matrix. Traits whose communalities (the proportion of variance in that trait explained by the principal component) were below 0.5 were removed from the analysis and a second PCA was run on the remaining traits (Johnson \& Wichern, 2002). The scree plot was used to determine how many components to retain. The resulting principal component (PC) scores for the four reliably rated lions were used in further analyses investigating the relationship between PC scores and GCM levels. While it is not ideal to utilize PCA to reduce the dimensionality of a dataset with a high variable to sample size ratio, as we have, we believe that if the resultant principal components are biologically relevant, this method is superior to more rudimentary univariate analyses that fail to incorporate all of the personality traits simultaneously and thus, result in multiple correlations requiring the use of Bonferroni corrections (Peres-Neto, 1999). Furthermore, while the general rule for utilizing PCA requires large sample sizes $(n>100)$, it has been argued that if the data are reliable, the factors are well-defined, and the communalities are high, PCA is a valid and useful technique for small sample sizes (Budaev, 2010).

Investigating the relationship between behavior and glucocorticoid metabolite levels. Linear mixed effects models investigated the impact of PC score, condition, and sex as fixed effects and ID as a random effect on log-transformed GCM levels ( $n=4$ lions). In order to assess the importance of ID as a random effect, the full model was compared to a model omitting the random effect by computing the likelihood ratio test statistic and its corresponding $p$-value. Significance of each fixed effect was assessed using analysis of variance to compare the full model to the model omitting each fixed effect.

To explore the existence of coping styles that link personality traits to physiological stress response (PC1) scores were regressed on the log-transformed GCM values $(n=4)$. To investigate coping styles further, the following variables were calculated using raw GCM values for all six lions: average, minimum, and maximum GCM (baseline, construction, or new habitat) as well as differences between these values across conditions (baseline to construction, construction to new habitat, and baseline to new 
habitat). Spearman's rank correlations were then used to investigate the relationship between these GCM values and average trait values (Siegel \& Castellan, 1988).

All statistics were run using $\mathrm{R}$ version 3.0.3 with the exception of the principal component analysis, which was run in JMP version 9.0 (R Core Team, 2013).

\section{Results}

\section{Glucocorticoid Metabolite Levels}

While analysis of variance revealed a significant effect of condition on GCM levels for all six lions overall, with a $26 \%$ reduction from baseline GCM levels to levels in the new habitat $\left(F_{2,224}=4.01, p\right.$ $=0.02$ ), the addition of ID as a random effect improved the fit of the model (LRT $=65.37, p<0.0001$ ), indicating that not all lions experienced the same effects of condition on GCM levels. Analysis of variance on each lion revealed that only Percival and Katie showed significantly different GCM values across conditions. Percival had a 97\% increase in average GCM from baseline to construction that fell back to baseline levels in the new habitat $\left(F_{2,27}=11.11, p=0.0003\right.$, Figure 1). Katie's GCM levels were $39 \%$ lower in the new habitat when compared to baseline levels, with no change during construction $\left(F_{2,41}\right.$ $=4.12, p=0.02$, Figure 1 ).

Validation of glucocorticoid levels as a measure of stress. Percival's highest GCM levels occurred in conjunction with a bout of pacing while he was inside during construction (Figure 2). Similarly, Katie showed higher than average levels the day after she was observed pacing. These were the only two lions who exhibited changes in GCM levels across conditions. As neither animal paced regularly, these results suggest that increased GCM levels likely reflect an animal that is experiencing negative stress or reduced welfare. Furthermore, Bikira had unusually high GCM levels after a blood draw and a veterinary examination, but did not have significantly different GCM levels across conditions, thus suggesting that the changes seen in Katie and Percival are a reflection of the mechanism these two lions used to cope with their environment.

\section{Personality Results}

Only 11 out of 18 behavioral traits passed IOR testing. After removing the seven unreliable traits, only four out of six lions passed IOR testing, thus confirming the need to test reliability on both traits AND individuals, as testing on only one component does not account for variation in the other (Table 2). For those 11 traits, concordance coefficients ranged from $\mathrm{W}=0.64$ to $\mathrm{W}=0.83$ with $p$-values between 0.03 and 0.09 . Of the four lions whose ratings passed reliability, concordance coefficients ranged from $\mathrm{W}$ $=0.696$ to 0.939 with $p$-values between 0.002 and 0.02 . 
Torgerson-White \& Bennett 237

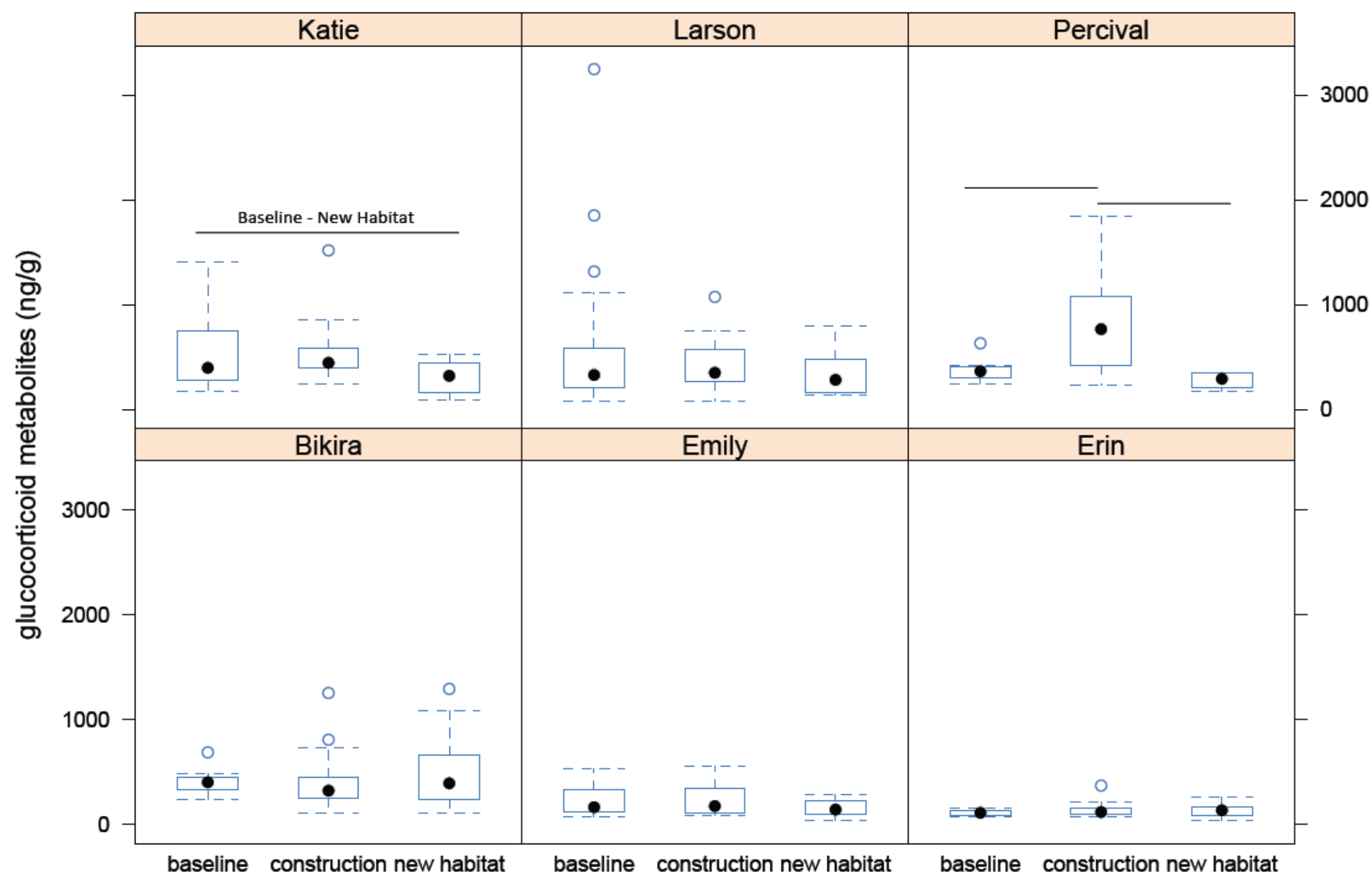

Figure 1. Glucocorticoid metabolite levels by lion. Horizontal lines above plots indicate differences in the mean at $p<0.05$. The black circles represent the medians. The box represents the $26^{\text {th }}$ and $75^{\text {th }}$ quartiles and the whiskers extend to the most extreme data point that is less than $1.5 *$ inner quartile range outside the box. 
Percival's fecal glucocorticoids over time (ng/g)

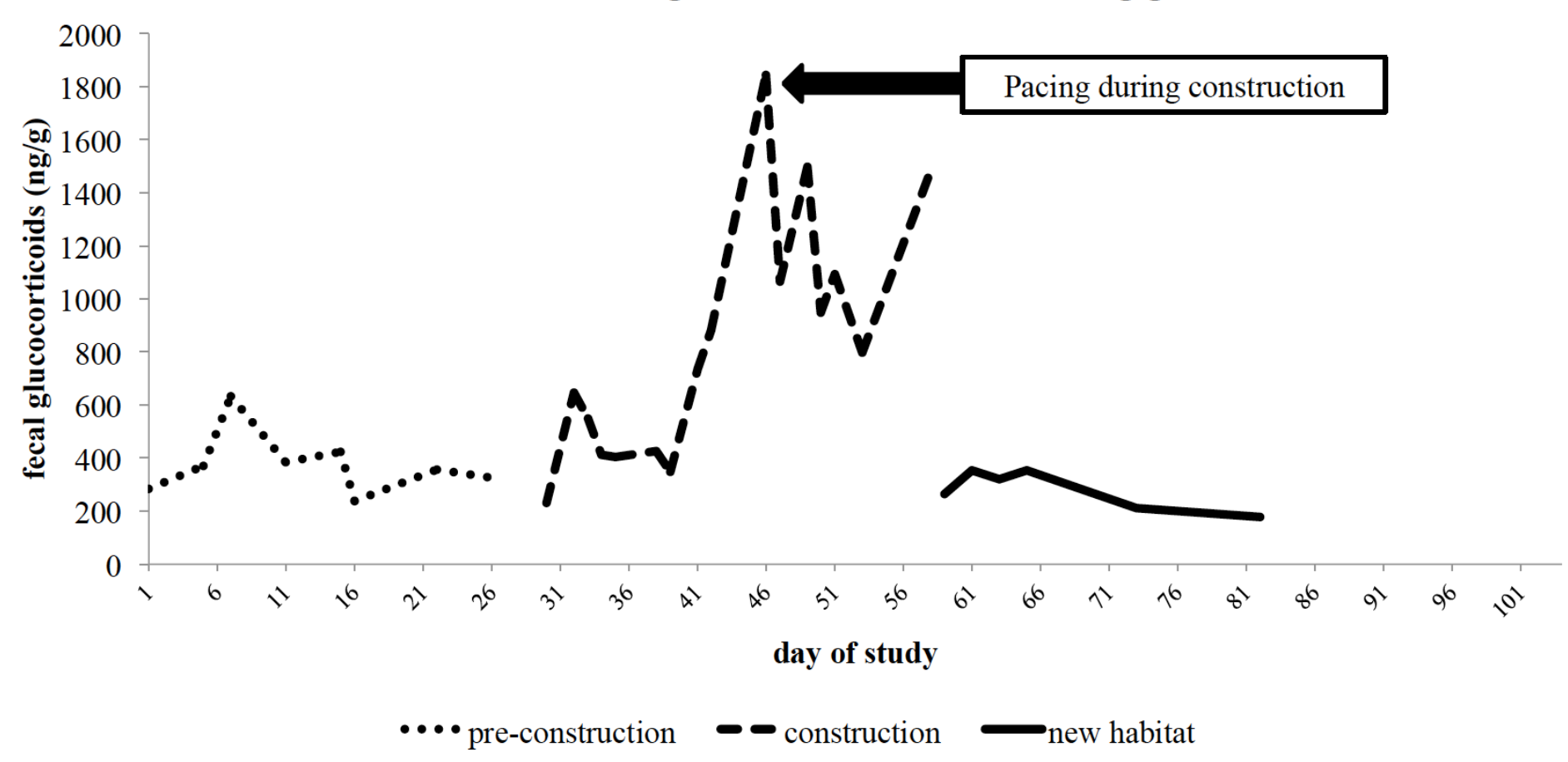

Figure 2. Percival's GCM values over time 
Table 2

Kendall's Coefficient of Concordance, W, and Associated p-values

\begin{tabular}{|c|c|c|}
\hline Trait & $\mathbf{W}$ & $p$ \\
\hline Curious & 0.827 & 0.0297 \\
\hline Friendly towards people & 0.822 & 0.0305 \\
\hline Insecure & 0.822 & 0.0305 \\
\hline Aggressive towards people & 0.763 & 0.043 \\
\hline Tense & 0.763 & 0.0433 \\
\hline Solitary & 0.708 & 0.0595 \\
\hline Self-assured & 0.699 & 0.0627 \\
\hline Vocal & 0.692 & 0.065 \\
\hline Smart & 0.647 & 0.084 \\
\hline Playful & 0.644 & 0.0853 \\
\hline Active & 0.635 & 0.09 \\
\hline Eccentric & 0.591 & 0.115 \\
\hline Fearful of conspecifics & 0.526 & 0.163 \\
\hline Fearful of people & 0.513 & 0.174 \\
\hline Excitable & 0.327 & 0.428 \\
\hline Friendly towards conspecifics & 0.32 & 0.441 \\
\hline Calm & 0.307 & 0.465 \\
\hline $\begin{array}{l}\text { Aggressive towards } \\
\text { conspecifics }\end{array}$ & 0.289 & 0.502 \\
\hline \multicolumn{3}{|l|}{ Lions } \\
\hline Katie & 0.939 & 0.0017 \\
\hline Erin & 0.848 & 0.00457 \\
\hline Larson & 0.800 & 0.00754 \\
\hline Bikira & 0.696 & 0.0219 \\
\hline Percival & 0.464 & 0.177 \\
\hline Emily & 0.245 & 0.693 \\
\hline
\end{tabular}

The communalities from the first PCA revealed that the traits curious and vocal should be removed from this analysis (Table 3). The principal component analysis containing the nine traits with communalities greater than 0.5 for the four lions who passed reliability revealed one principal component axis (PC). PC1 had an eigenvalue of 7.74 and explained $86 \%$ of the variance in the data and represents a sociability/neuroticism axis. The traits self-assured, friendly towards people, smart, active, and playful loaded strongly and positively into PC1 while the traits tense, solitary, insecure, and aggressive towards people loaded strongly and negatively (Table 3). Hence, animals with larger PC1 scores were more social and less neurotic and those with smaller scores were more neurotic and more anti-social. 
Table 3

Loading Values and Communalities for Principal Component

\begin{tabular}{lccc}
\hline & Initial Communality & PC1 & Final Communality \\
\hline Self-assured & 0.971 & 0.983 & 0.967 \\
Friendly towards people & 0.870 & 0.949 & 0.901 \\
Smart & 0.839 & 0.930 & 0.865 \\
Active & 0.860 & 0.904 & 0.817 \\
Playful & 0.543 & 0.705 & 0.497 \\
Aggressive towards people & 0.797 & -0.916 & 0.838 \\
& & & \\
Insecure & 0.900 & -0.963 & 0.927 \\
Solitary & 0.964 & -0.970 & 0.940 \\
Tense & 0.972 & -0.994 & 0.988 \\
Curious & 0.370 & $N A$ & $N A$ \\
Vocal & 0.190 & $N A$ & $N A$ \\
\hline
\end{tabular}

\section{The Link Between Personality and Glucocorticoids}

Linear regression revealed that lions that are more social and less neurotic (higher PC1 scores) have lower GCM levels and those that are more anti-social and more neurotic (lower PC1 scores) have higher GCM levels $\left(F_{1,159}=10.64, p=0.001\right)$.

Linear and linear mixed effect models revealed that the only significant predictor of GCM levels is ID $\left(F_{3,157}=26.27, p<0.0001\right)$. The inclusion of ID as a random effect in a model with PC1, condition, and sex as fixed effects significantly improved the fit of the model (Table 4), but no other variables were found to have an impact on the fit of the full model.

Table 4

Linear mixed effects models predicting glucocorticoid metabolite levels using in the four reliably rated lions

Model Fixed Effects $\quad$ Random Effects $\quad$ Least Ratio Test Statistic/ $\chi^{2} \quad$ p - value $\quad$ AIC

\begin{tabular}{llllll}
\hline Full & PC1, Condition, Sex & ID & & & 77 \\
1 & PC1, Condition, Sex & & 19.90 & $p<0.0001$ & 95 \\
2 & PC1, Condition & ID & 0.32 & 0.57 & 74 \\
3 & PC1, Sex & ID & 2.58 & & \\
4 & Condition, Sex & ID & 1.16 & 0.28 & 67 \\
5 & PC1, Sex & Condition|ID & 3.30 & 0.28 & 71 \\
6 & PC1 & ID & 2.93 & 0.35 & 74 \\
7 & Condition & ID & 1.43 & 0.40 & 65 \\
8 & Sex & ID & 3.68 & 0.49 & 69 \\
\hline
\end{tabular}


Analysis of all traits individually revealed that the traits active and vocal (vocal was omitted from the principal component analysis) were linked with GCM values. Spearman's rank correlations between the average trait values for the six lions and the GCM measures revealed that the most vocal lions had the highest minimum GCM levels in the old habitat (rho $=0.83, p=0.04$, Table 5), and the highest average and maximum GCM levels when kept inside during construction (rho $=0.89$ and $0.94, p=0.02$ and 0.004, respectively). Animals that were most active (excluding abnormal repetitive behavior), however, had the lowest minimum GCM levels in the old habitat (rho $=-0.89, p=0.02$ ), and the lowest average and maximum GCM levels in the new habitat (rho $=-0.83$ and $-0.83, p=0.04$ and 0.04 , respectively). Because Percival was one of only two lions who showed significant differences in GCM levels across conditions (and he would have been left out of the analysis if we used only the four reliably-rated lions), and because Spearman's rank correlations cannot be performed on a sample size less than six, we chose to include all six lions in these analyses. For this reason, results should be considered speculative.

Table 5

Spearman's rank correlations linking the traits active and vocal to glucocorticoid metabolite levels, $n=6$ lions

\begin{tabular}{llll}
\hline Trait & GCM measure & Spearman's rho & p - value \\
\hline vocal & minimum baseline & 0.83 & 0.04 \\
vocal & mean construction & 0.89 & 0.02 \\
vocal & maximum construction & 0.94 & 0.004 \\
active & minimum baseline & -0.89 & 0.02 \\
active & mean new habitat & -0.83 & 0.04 \\
active & maximum new habitat & -0.83 & 0.04 \\
\hline
\end{tabular}

\section{Why Weren't All the Lions Rated Reliably?}

After performing IOR on the lions and discovering that two of the lions (Percival and Emily) did not pass reliability, we decided to examine post hoc what might be driving these results. We tested three hypotheses based on the numbers of extreme trait ratings (ratings below 20 or above 100) that each caretaker gave and that each lion received (Figure 3). The first hypothesis stated that the lions' history at the zoo resulted in some being rated more extremely than others. To test the first hypothesis, we examined the correlation between the number of extreme trait ratings and the number of years that the lion had been at the zoo (Spearman's rho $=0.12, p=0.82$ ). The second hypothesis explored the possibility that one of the caretakers was rating the lions more extremely than the others, perhaps because he or she had different responsibilities with regard to caring for the lions, and thus experienced different facets of their personalities. To test the second hypothesis, a Pearson's chi-squared test investigated differences in the number of extreme ratings by caretaker and revealed no significant differences (caretaker; $\chi_{4}^{2}=6, p=0.20$ ). We also visually inspected a bar graph that depicted the number of extreme rating that each caretaker gave each lion (Figure 4). The third hypothesis, which stems from the idea that ratings do not account for behavioral plasticity, predicted that unreliably rated lions behaved differently depending on which keeper was present (Réale \& Dingemanse, 2012). To test the third hypothesis, a Pearson's chi-squared test investigated differences in the number of extreme ratings by lion and revealed no significant differences $\left(\chi_{25}^{2}=30, p=0.2\right)$, but a bar graph revealed that Percival and Emily, the two lions who were not reliably rated, were given the least number of extreme trait ratings (Figure 3 ). We then tested the correlation between the number of extreme trait ratings and Kendall's coefficient of concordance, $\mathrm{W}$, and found that the two were strongly and positively correlated, thus suggesting that the less reliably rated lions do have less extreme personalities (Spearman's rho $=0.89, p=0.03$ ). 


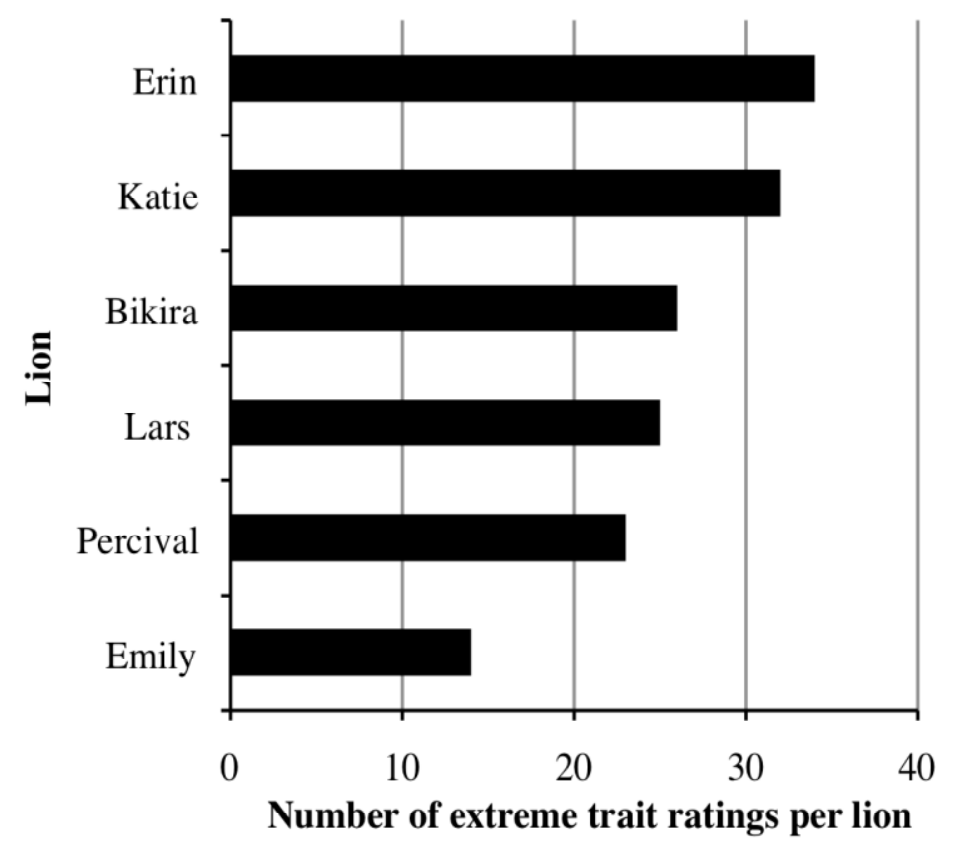

Figure 3. The number of extreme trait ratings given to each lion

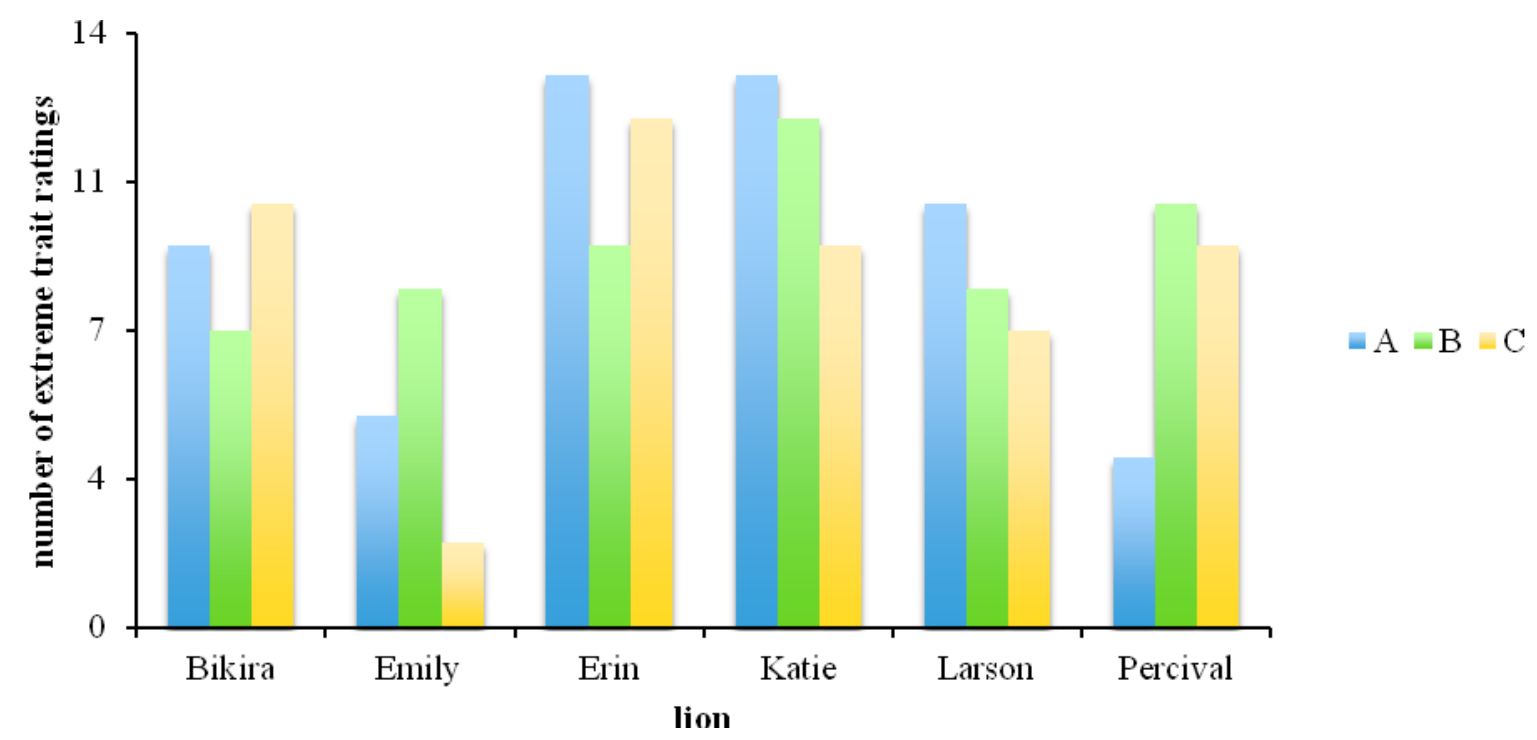

Figure 4. The number of extreme trait ratings given to each lion by each keeper (A, B, and C)

\section{Discussion}

The original goal of this study was to discern coping styles in the African lions at the Detroit Zoo by investigating the relationship between fecal glucocorticoid metabolites and personality, thus creating a tool that could be used in the future to inform the individual management of each animal. Glucocorticoid metabolite levels for the lions revealed that four out of the six lions showed no difference in GCM levels 
across conditions, but that Percival may have struggled while inside during construction and Katie's welfare may have improved in the new habitat.

\section{Lion Personality and its Relationship with Glucocorticoid Metabolites}

Previous studies have uncovered similar personality structure across species within the family Felidae. Personality traits observed most often include activity or extraversion, sociability, neuroticism, and aggression (Gartner \& Weiss, 2013). Our study uncovered a single personality axis that combines many of the traits present in previously described sociability and neuroticism axes and was negatively correlated with the neuroticism factor uncovered in Gartner et al.'s study of personality structure in African lions (congruence coefficient $=-0.873$, Gartner et al., in press; Gartner \& Weiss, 2013; LorenzoSeva \& Ten Berge, 2006). Most often in the animal personality literature, a personality axis reflects behavioral traits related to a single personality axis rather than two, as we have found here. This finding suggests that perhaps in these lions, sociability and neuroticism are linked and may form a behavioral syndrome (Réale \& Dingemanse, 2012; Sih, Bell, \& Johnson, 2004). The traits friendly and aggressive towards people allude to the level of sociability with regards to humans while the traits insecure, tense, solitary, self-assured, and smart explain the degree of a trait referred to as neurotic in lions. Neuroticism is a trait that stems from the Five Factor Model in human psychology and has been associated with passive and inefficient coping mechanisms (Goldberg, 1990; Watson \& Hubbard, 1996). Understanding the levels of sociability and neuroticism of each individual lion can inform management decisions when the lions are exposed to potential stressors (e.g., new social situations, habitat changes, veterinary procedures, etc.). For instance, in this study, the new habitat allowed decreased proximity between the lions and the visitors. Lions that scored negatively on the sociability-neuroticism axis were more anti-social and more neurotic. As neuroticism has been linked with poor coping abilities in humans and neurotic lions were more anti-social, it is likely that the welfare of these particular animals may have benefitted if given a gradual and carefully orchestrated transition to the new habitat and if care is taken to ease the transition to any new situation, social or otherwise.

While our sample size was too small to uncover an actual coping style that related the sociabilityneuroticism axis to HPA axis reactivity (rather than activity), we did find that being more vocal was associated with having higher baseline GCM values as well as higher GCM values when the animals were inside during construction. Conversely, being more active was associated with lower baseline GCM levels and lower levels in the new habitat. These two behaviors could represent traits already reported and related to impulsiveness in African lions (Gartner et al., in press). Perhaps lions that didn't cope as well were generally more vocal in response to stressful events rather than actively exploring the new environment. Conversely, lions that coped well may have been more willing to move about and explore the new habitat. It should be noted that none of our traits correlated significantly with any of the changes in GCM levels from one condition to another. Although these results are largely speculative, they do highlight the need not only for more research on this topic, but specifically for multi-institutional studies that would give rise to larger sample sizes and hence, more rigorous statistical analysis, a conclusion also supported by the non-significant models attempting to link personality, condition, and sex to GCM levels.

\section{Rating Methodology and Behavioral Plasticity}

While the rating method is easier to use than behavioral coding, it has shortcomings. First and foremost, it is difficult and time-consuming to create surveys that measure exactly what one hopes to measure (Watters \& Powell, 2012). Second, inter-observer reliability must be measured to ensure that the observers agree on the animal's personality traits. In other words, the survey must be both valid and reliable (Carter, Feeney, Marshall, Cowlishaw, \& Heinsohn, 2013). As demonstrated by this case study and as seen in other studies, reliability analyses of caretaker ratings often result in low reliability scores. This results in certain portions of the survey, entire animals, or entire observers being removed from the analysis (Highfill et al., 2010; Phillips \& Peck, 2007; Powell \& Svoke, 2008; Wielebnowski, 1999). 
Unfortunately, these data may contain valid information about how an animal adapts its behavior to the present situation. Furthermore, by averaging across observers prior to loading the ratings into a principal component analysis, more information is lost regarding how an animal responds to different situations such as the presence of different caretakers. It is of note, that in this study, none of the traits that measured how a lion dealt with conspecifics was reliably rated while traits that relate the lion to humans were usually reliably rated. Perhaps we can better assess how animals react to humans by using a simple rating, but we need to collect behavioral data regarding how an animal behaves with regard to its conspecifics.

After we found that two of the lions didn't pass reliability testing, we tested three hypotheses that might explain these results (see Results). We tested and rejected the first hypothesis by looking for a positive correlation between the number of extreme trait ratings and the number of years at the zoo, thus investigating whether more time spent with a lion would lead to more chances to observe extreme behavior. We tested and rejected the second hypothesis by looking for differences in the number of extreme ratings that caretakers gave each lion and finding that there wasn't a consistent trend for one of the keepers to rate the lions more extremely.

We tested and accepted the third hypothesis by investigating the number of extreme trait ratings that each lion received and finding that the two unreliably rated lions had the least extreme personalities. Previous research on behavioral plasticity has revealed that animals with extreme personalities are less likely to exhibit plasticity while those whose personalities fall in the mid-range exhibit the most plasticity (West-Eberhard, 1989). For instance, among mice, the most aggressive individuals do not adjust their levels of aggression, whereas those who are less aggressive vary their aggression levels depending on the context (Natarajan, de Vries, Saaltink, De Boer, \& Koolhaas, 2009). This tendency may explain the variation in personality ratings for these two lions. They were rated the least extremely, had the largest variation in behavioral ratings given by the caretakers, and thus may have exhibited the highest degree of plasticity.

The concept of behavioral plasticity seems in conflict with the concept of animal personality, which is defined as behavioral consistency across time and contexts (Gosling \& John, 1999; Réale et al., 2007; Sih, Bell, Johnson, et al., 2004). However, the ability to adjust behavior to the current situation contributes to survival in certain environments, hence plasticity as a dimension of personality should persist in a population (Piersma \& Drent, 2003; Réale \& Dingemanse, 2010). Dingemanse, Kazem, Réale, $\&$ Wright (2010) resolve this conflict arguing that, while the expression of an individual's personality may remain relatively consistent within a population, there is variation in behavior within the individual. They crafted the concept of behavioral reaction norms, a "set of behavioral phenotypes that a single individual produces in a given set of environments", that incorporate both the magnitude of a trait as well as the degree of plasticity. In this study, rating methodology succeeded in measuring the magnitude of a behavioral trait. But as Réale and Dingemanse (2012) pointed out, the study was not designed to incorporate any element of plasticity. Ignoring information pertaining to the ability (or inability) of an animal to tailor its behavior to its current situation may leave us with an inaccurate conclusion regarding how well an animal can cope in captivity.

\section{The Human-Animal Relationship as a Measure of Behavioral Plasticity}

Studies with farm, lab and zoo animals indicate that the relationships between a caretaker and the animals in his or her care are unique. In 2013, Geoff Hosey proposed a model of how animals could interpret these relationships, with the caretaker being seen as a friend or enemy, as part of the inanimate environment, as a conspecific, and/or as a source of stimulation. Further, the presence of humans, and thus the nature of the human-animal relationship, has been demonstrated to impact behavior (Davey, 2007; Fernandez, Tamborski, Pickens, \& Timberlake, 2009; Hosey, 2000; 2005; 2008; 2013). In this study, several personality traits, and two lions, were excluded from the analyses because the caretakers each had their own, very distinct, ideas of how the lions rated on the behavioral traits. Whereas traditionally, disagreement like this amongst caretakers might be viewed as human error, we propose that, despite the differences in their ideas of the lions' personalities, each caretaker may have been correct. 
While caretaker error is always a possibility, variation could also be the result of an animal varying its behavior depending on which caretaker is present. This variation could reflect an animal's history and experiences with a particular person (or similar persons) and thus be indicative of the level of behavioral plasticity present in that animal - a component of behavior that is integral to coping with environmental stressors and important in determining the level of welfare that an animal experiences in captivity.

\section{Future Directions}

From the perspective of personality studies on captive animals, there are three ways to remedy this loss of important information regarding plasticity. Studies that use rating methodology could use all of the caretaker ratings without measuring reliability, thus assuming that all variation is due to plasticity. However, this methodology would be unable to uncover actual error in caretaker ratings. Alternatively, as suggested by Dingemanse et al. (2010), a plasticity measure could be incorporated into the personality surveys. For instance, keepers could be asked to repeatedly rate the animals with regard to the same traits on different occasions (e.g., before and after construction), thus recording plasticity by examining differences in behavior across contexts. Unfortunately, this method still doesn't account for the plasticity that manifests itself in how the animal acts in the presence of different caretakers. Finally, it has been demonstrated that certain personality traits can be reliably and validly rated by inexperienced observers (Petelle \& Blumstein, 2014). This method is superior in that the relationship of the raters with the animal is no longer a confounding factor. In order to account for plasticity, further research should examine the feasibility of having inexperienced observers watch the animals in different contexts, and then rate them in each context separately.

Reliable measures of behavioral plasticity in captive animals would provide us with unique opportunities to investigate the impacts of plasticity on fitness and welfare at the individual level as well as success in captivity at the species level. Whereas the stressors of captivity are different from those that animals experience in the wild, many captive stressors are the same across species (e.g., spatial compression at night and/or during the winter, reduced choice with regard to social partners, etc.). Research is needed that examines the ecological and evolutionary processes behind the divergence of coping mechanisms (Réale \& Dingemanse, 2012). Multi-species comparisons of behavioral plasticity in captive animals may help us to understand the role of plasticity in the evolution of coping mechanisms across species while simultaneously elucidating the role of plasticity as a dimension of personality and thus, providing guidance for better captive management of individuals.

\section{Conclusion}

The goal of this study was to validate a method by which animal caretakers could easily and objectively predict how an animal might cope with an environmental stressor, thus allowing for individualized animal management decisions during potentially stressful times. Whereas we did not accomplish this goal in its entirety, we did uncover a sociability-neuroticism axis that was linked to GCM levels and discussed how vocalizing and being active may be related to coping. This study also revealed the limitations of rating methodology when being used to assess animal personality. This methodology is useful and reliable for animals who behave the same way in every situation. However, caretaker rating methodology fails to account for a key component of behavior, how an animal might respond differently to different situations-an evolutionarily important coping strategy that likely contributes to the level of welfare than an animal experiences in captivity. Finally, this study makes evident the need for more multi-institutional studies investigating how personality impacts individual animal welfare.

\section{Acknowledgements}

We would like to thank the carnivore caretakers, especially Cindy Colling, Melanie Hiam, and John Wojciechowski for their cooperation in this study. We would also like to thank Stephanie Allard and 
Scott Carter for their comments on this manuscript. Finally, we would like to thank three anonymous reviewers for their astute comments and suggestions that only served to strengthen this paper.

\section{References}

Armstrong, D. M., \& Santymire, R. M. (2013). Hormonal and behavioral variation in pied tamarins housed in different management conditions. Zoo Biology, 32, 299-306. doi: 10.1002/zoo.21023

Association of Zoos and Aquariums Animal Welfare Committee. (2014). Animal Welfare Committee, Retrieved from http://www.aza.org

Biro, P. A., \& Stamps, J. A. (2008). Are animal personality traits linked to life-history productivity? Trends in Ecology \& Evolution, 23, 361-368.

Brown, J. L., Kersey, D. C., Freeman, E. W., \& Wagener, T. (2010). Assessment of diurnal urinary cortisol excretion in Asian and African elephants using different endocrine methods. Zoo Biology, 29, 274-283. doi: 10.1002/zoo.20268

Budaev, S. V. (2010). Using principal components and factor analysis in animal behaviour research: Caveats and guidelines. Ethology, 116, 472-480.

Budaev, S. V., Zworykin, D. D., \& Mochek, A. D. (1999). Individual differences in parental care and behaviour profile in the convict cichlid: A correlation study. Animal Behaviour, 58, 195-202.

Burgener, N., Gusseta, M., \& Schmidb, H. (2008). Frustrated appetitive foraging behavior, stereotypic pacing, and fecal glucocorticoid levels in snow leopards (Uncia uncia) in the Zurich zoo. Journal of Applied Animal Welfare Science, 11, 74-83. doi: 10.1080/10888700701729254

Carter, A., Feeney, W., Marshall, H., Cowlishaw, G., \& Heinsohn, R. (2013). Animal personality: What are behavioural ecologists measuring? Biological Reviews, 88, 465-475.

Cheek, J. M., \& Buss, A. H. (1981). Shyness and sociability. Journal of Personality and Social Psychology, 41, 330.

Coleman, K. (2012). Individual differences in temperament and behavioral management practices for nonhuman primates. Applied Animal Behaviour Science, 137, 106-113. doi: 10.1016/j.applanim.2011.08.002

Davey, G. (2007). Visitors' effects on the welfare of animals in the zoo: A review. Journal of Applied Animal Welfare Science, 10, 169-183. doi: 10.1080/10888700701313595

Dingemanse, N. J., Kazem, A. J. N., Réale, D., \& Wright, J. (2010). Behavioural reaction norms: Animal personality meets individual plasticity. Trends in Ecology \& Evolution, 25, 81-89. doi: 10.1016/j.tree.2009.07.013

Dingemanse, N. J., \& Réale, D. (2005). Natural selection and animal personality. Behaviour, 142, 9-10.

Faraway, J. J. (2006). Extending the linear model with R: Generalized linear, mixed effects and nonparametric regression models. Boca Raton, FL: Chapman \& Hall/CRC.

Fernandez, E. J., Tamborski, M. A., Pickens, S. R., \& Timberlake, W. (2009). Animal-visitor interactions in the modern zoo: Conflicts and interventions. Applied Animal Behaviour Science, 120, 1-8. doi: 10.1016/j.applanim.2009.06.002

Gartner, M. C., Powell, D. M., \& Weiss, A. (in press). Personality structure in the domestic cat (Felis silvestris catus), Scottish wildcat (Felis silvestris grampia), clouded leopard (Neofelis nebulosa), snow leopard (Panthera uncia), and African lion (Panthera leo): A comparative study. Journal of Comparative Psychology.

Gartner, M. C., \& Weiss, A. (2013). Personality in felids: A review. Applied Animal Behaviour Science, 144, 1-13. doi: http://dx.doi.org/10.1016/j.applanim.2012.11.010

Goldberg, L. R. (1990). An alternative "description of personality": The big-five factor structure. Journal of Personality and Social Psychology, 59, 1216.

Gosling, S. D., \& John, O. P. (1999). Personality dimensions in nonhuman animals: A cross-species review. Current Directions in Psychological Science, 8(3), 69-75.

Highfill, L., Hanbury, D., Kristiansen, R., Kuczaj, S., \& Watson, S. (2010). Rating vs. coding in animal personality research. Zoo Biology, 29, 509-516. doi: 10.1002/zoo.20279

Hosey, G. (2000). Zoo animals and their human audiences: What is the visitor effect? Animal Welfare, 9, 343-357.

Hosey, G. (2005). How does the zoo environment affect the behaviour of captive primates? Applied Animal Behaviour Science, 90, 107-129. doi: 10.1016/j.applanim.2004.08.015

Hosey, G. (2008). A preliminary model of human-animal relationships in the zoo. Applied Animal Behaviour Science, 109, 105-127. doi: 10.1016/j.applanim.2007.04.013

Hosey, G. (2013). Hediger revisited: How do zoo animals see us? Journal of Applied Animal Welfare Science, 16, 338-359. 
Johnson, R. A., \& Wichern, D. W. (2002). Applied multivariate statistical analysis (5th ed.). Upper Saddle River, NJ: Prentice Hall.

Koolhaas, J. M., Korteb, S. M., Boer, S. F. D., Vegt, B. J. V. D., Van Reenen, C. G., Hopster, H., . . Blokhuis, H. J. (1999). Coping styles in animals - Current status in behavior and stress physiology. Neuroscience and Biobehavioral Reviews, 23, 925-935.

Loeding, E., Thomas, J., Bernier, D., \& Santymire, R. (2011). Using fecal hormonal and behavioral analyses to evaluate the introduction of two Sable antelope at Lincoln Park Zoo. Journal of Applied Animal Welfare Science, 14, 220-246. doi: 10.1080/10888705.2011.576968

Lorenzo-Seva, U., \& Ten Berge, J. M. (2006). Tucker's congruence coefficient as a meaningful index of factor similarity. Methodology: European Journal of Research Methods for the Behavioral and Social Sciences, 2, 57-64.

Mason, G. J. (2010). Species differences in responses to captivity: Stress, welfare and the comparative method. Trends in Ecology \& Evolution, 25, 713-721. doi: 10.1016/j.tree.2010.08.011

McDougall, P. T., Réale, D., Sol, D., \& Reader, S. M. (2006). Wildlife conservation and animal temperament Causes and consequences of evolutionary change for captive, reintroduced, and wild populations. Animal Conservation, 9, 39-48. doi: 10.1111/j.1469-1795.2005.00004.x

Natarajan, D., de Vries, H., Saaltink, D. J., De Boer, S. F., \& Koolhaas, J. M. (2009). Delineation of violence from functional aggression in mice: An ethological approach. Behavior Genetics, 39, 73-90.

Peres-Neto, P. R. (1999). How many statistical tests are too many? The problem of conducting multiple ecological inferences revisited. Marine Ecology Progress Series, 176, 303-306.

Petelle, M. B., \& Blumstein, D. T. (2014). A critical evaluation of subjective ratings: Unacquainted observers can reliably assess certain personality traits. Current Zoology, 60, 162-169.

Phillips, C., \& Peck, D. (2007). The effects of personality of keepers and tigers (Panthera tigris tigris) on their behaviour in an interactive zoo exhibit. Applied Animal Behaviour Science, 106, 244-258. doi: 10.1016/j.applanim.2007.01.007

Piersma, T., \& Drent, J. (2003). Phenotypic flexibility and the evolution of organismal design. Trends in Ecology \& Evolution, 18, 228-233.

Powell, D., \& Svoke, J. (2008). Novel environmental enrichment may provide a tool for rapid assessment of animal personality: A case study with giant pandas (Ailuropoda melanoleuca). Journal of Applied Animal Welfare Science, 11, 301-318. doi: 10.1080/10888700802329673

R Core Team. (2013). R: A language and environment for statistical computing R. F. f. S. Computing (Ed.) Retrieved from http://www.R-project.org

Réale, D., \& Dingemanse, N. J. (2010). Personality and individual social specialisation. In Social behaviour: Genes, ecology and evolution (pp. 417-440), New York, NY: Cambridge University Press.

Réale, D., \& Dingemanse, N. J. (July 2012). Animal personality. In: eLS. John Wiley \& Sons, Ltd: Chichester. doi: 10.1002/9780470015902.a0023570

Réale, D., Reader, S. M., Sol, D., McDougall, P. T., \& Dingemanse, N. J. (2007). Integrating animal temperament within ecology and evolution. Biological Reviews, 82, 291-318. doi: 10.1111/j.1469-185X.2007.00010.x

Shideler, S., Ortuno, A., Moran, F., Moorman, E., \& Lasley, B. (1993). Simple extraction and enzyme immunoassays for estrogen and progesterone metabolites in the feces of Macaca fascicularis during nonconceptive and conceptive ovarian cycles. Biology of Reproduction, 48, 1290-1298.

Siegel, S., \& Castellan, N. J. (1988). Nonparametric statistics for the behavioral sciences (2nd ed.). New York: McGraw-Hill.

Sih, A., Bell, A., \& Johnson, J. C. (2004). Behavioral syndromes: An ecological and evolutionary overview. Trends in Ecology \& Evolution, 19, 372-378.

Sih, A., Bell, A. M., Johnson, J. C., \& Ziemba, R. E. (2004). Behavioral syndromes - An integrative overview. The Quarterly Review of Biology, 79, 241-277. doi: 10.1086/422893

Vazire, S., Gosling, S. D., Dickey, A. S., \& Schapiro, S. J. (2007). Measuring personality in nonhuman animals. In R. W. Robins, R. C. Fraley, \& R. F. Krueger (Eds.), Handbook of research methods in personality psychology (pp. 190-206). New York: The Guilford Press.

Wasser, S. K., Hunt, K. E., Brown, J. L., Cooper, K., Crockett, C. M., Bechert, U., ...Monfort, S. L. (2000). A generalized fecal glucocorticoid assay for use in a diverse array of nondomestic mammalian and avian species. General and Comparative Endocrinology, 120, 260-275.

Watson, D., \& Hubbard, B. (1996). Adaptational style and dispositional structure: Coping in the context of the Five Factor model. Journal of Personality, 64, 737-774. 
Watters, J. V., \& Powell, D. M. (2012). Measuring animal personality for use in population management in zoos: Suggested methods and rationale. Zoo Biology, 31, 1-12. doi: 10.1002/zoo.20379

West-Eberhard, M. J. (1989). Phenotypic plasticity and the origins of diversity. Annual review of Ecology and Systematics, 249-278.

Whitham, J. C., \& Wielebnowski, N. (2009). Animal-based welfare monitoring: Using keeper ratings as an assessment tool. Zoo Biology, 28, 545-560. doi: 10.1002/zoo.20281

Wielebnowski, N. C. (1999). Behavioral differences as predictors of breeding status in captive cheetahs. Zoo Biology, 18, 335-349. doi: 10.1002/(sici)1098-2361(1999)18:4<335::aid-zoo8>3.0.co;2-x

Wolf, M., van Doorn, G. S., Leimar, O., \& Weissing, F. J. (2007). Life-history trade-offs favour the evolution of animal personalities. Nature, 447, 581-584. 